\title{
Characterization of the Transcriptome of Hair Cell Regeneration in the Neonatal Mouse Utricle
}

\author{
Jinghong Han ${ }^{a, b, c}$ Hanwei Wu ${ }^{b}$ Hongyi Hu Weiqiang Yang ${ }^{a}$ Hongsong Dong ${ }^{c}$ \\ Yuchen Liu ${ }^{b}$ Guohui Nie \\ aDepartment of Otolaryngology, Peking University Shenzhen Hospital, Shenzhen, 'Institute of \\ Translational Medicine, Shenzhen Second People's Hospital, The First Affiliated Hospital of Shenzhen \\ University, Shenzhen, 'Department of Otolaryngology, The First Affiliated Hospital of Shenzhen \\ University, Shenzhen, China
}

\section{Key Words}

Hair cell $\bullet$ Mouse utricle $•$ Neomycin $•$ RNA-Seq $・$ zf-C2H2

\begin{abstract}
Background/Aims: Hearing and balance deficits are mainly caused by loss of sensory inner ear hair cells. The key signals that control hair cell regeneration are of great interest. However, the molecular events by which the cellular signals mediate hair cell regeneration in the mouse utricle are largely unknown. Methods: In the present study, we investigated gene expression changes and related molecular pathways using RNA-seq and qRT-PCR in the newborn mouse utricle in response to neomycin-induced damage. Results: There were 302 and 624 genes that were found to be up-regulated and down-regulated in neomycin-treated samples. GO and KEGG pathway analyses of these genes revealed many deregulated cellular components, molecular functions, biological processes and signaling pathways that may be related to hair cell development. More importantly, the differentially expressed genes included 9 transcription factors from the $\mathrm{zf}-\mathrm{C} 2 \mathrm{H} 2$ family, and eight of them were consistently down-regulated during hair cell damage and subsequent regeneration. Conclusion: Our results provide a valuable source for future studies and highlighted some promising genes, pathways or processes that may be useful for therapeutic applications.
\end{abstract}

\section{Introduction}

Hearing and balance deficits are major health problems over the world, and they are mainly caused by loss of sensory inner ear hair cells [1]. Hair cells are recognized as primary transducers for perception of sound and balance [2]. In the sensory epithelium, there are also supporting cells that contact the basolateral surfaces of hair cells and mediate hair cell

Yuchen Liu

and Guohui Nie
Institute of Translational Medicine, Shenzhen Second People's Hospital

The First Affiliated Hospital of Shenzhen University, Shenzhen 518035 (China)

E-Mail s_ycliu2007@126.com; nghui@21cn.com 
survival and death [3]. The sensory hair cells are susceptible to damage, and their damage is often caused by noise exposure, viral or bacterial infections, aging, and ototoxic drugs [4]. In sharks, bony fish, amphibians, reptiles, and birds, hair cell damage stimulates the surrounding supporting cells to acquire the hair cell phenotype, thus leading to spontaneous regeneration of hair cells [5]. However, the loss of hair cells is permanent in mature mammals. Only supporting cells in newborns may have a limited capacity to reproduce hair cells [6]. It has been reported that the supporting cells in mammalian ears develop unique characteristics as they mature, which block regenerative responses to hair cell loss [7].

The key genes that control cell regeneration in neonatal mouse inner ear are of great biological and clinical interest. For example, Notch, FGF, Wnt and BMP signalings and the transcription factors Atoh1 and Sox2 have been linked to hair cell regeneration in recent investigations [8-10]. Despite various alternative mechanistic hypotheses were proposed to account for the limits to hair cell regeneration in mammals over the past years [11], the precise molecular mechanism that initiates hair cell regeneration remains largely unclear. To better understand the signaling events of hair cell regeneration after damage, investigating the transcriptional profiles of regenerating hair cells should be the first step. By using RNAseq, two previous studies have investigated the changes in the transcriptional profiles during hair cell regeneration in the mouse cochlea $[12,13]$. The high degree of differentiation and specialization of hair cells in the mammalian cochlea suggests that regeneration may be limited in this area, but hair cells in the vestibular system of mammals are most likely to regenerate due to their similarity to their counterparts in non-mammals. However, the molecular events by which the cellular signals mediate hair cell regeneration in the vestibular system including utricle are still unknown.

To address the above issue, in the present study, we revealed gene expression changes and related molecular pathways in the newborn mouse utricle in response to damage induced by neomycin, the most commonly used hair cell damage inducer. These results provide a new resource for fully understanding the detailed molecular mechanisms of hair cell regeneration in utricle. An understanding of such mechanisms will be important for efforts to learn how to release the mammalian supporting cells' potential to reproduce hair cells.

\section{Materials and Methods}

\section{Animals}

All experiments involving animals were housed and handled in accordance with protocols approved by the Institutional Review Board of Shenzhen University. Newborn C57Bl/6 mice were obtained from the Animal Center of the Academy of Sciences.

Utricle dissection and neomycin injury in the neonatal mouse utricle

Postnatal day (P)2 mice were sacrificed, and the utricles from mice were dissected out and grown in DMEM/F12 medium supplemented with N2 (1:100 dilution, Gibco), B27 (1:50 dilution, Gibco), and ampicillin $(50 \mu \mathrm{g} / \mathrm{ml}$, EMD millipore). At $7 \mathrm{~h}$ after the beginning of the culture, the utricles were treated with $3 \mathrm{mM}$ neomycin (Sigma) or normal medium for $24 \mathrm{~h}$. Then, neomycin was removed, and the utricles were grown for $3 \mathrm{~d}$ in normal medium.

\section{Immunohistochemistry}

The utricles were fixed for $30 \mathrm{~min}$ at room temperature with $4 \%$ paraformaldehyde in $0.1 \mathrm{M}$ phosphate buffer and then thoroughly rinsed with $0.01 \mathrm{M}$ PBS. After being treated in $0.01 \mathrm{M}$ PBS containing $1 \%$ Triton $-100 \mathrm{X}$ (PBST) and 10\% fetal bovine serum for $1 \mathrm{~h}$ at room temperature, the specimens were blocked the mouse monoclonal antibody against Myo7a (1:200 dilution; Santa Cruz Biotechnology) and the rabbit polyclonal antibody against Sox2 (1:500 dilution; EMD millipore) at $4{ }^{\circ} \mathrm{C}$ overnight. Appropritate Alexaconjugated secondary antibodies were used for detection. The proliferating cells were labeled with EdU (Click-iT EdU Alexa Fluor 647 imaging kit, Invitrogen) for 40 min according to the manufacturer's protocol. 
Table 1. Primers of genes used in RT-qPCR

\begin{tabular}{lcc}
\hline Gene & Forward Primer & Reverse Primer \\
\hline$\beta$-actin & GGCTGTATTCCCTCCATCG & CCAGTTGGTAACAATGCCATGT \\
GM22 & CAGCGCGTCTACCTGTGTC & CCTCCTGATGACGTGCATGA \\
Zfp474 & CACAAGGAACTATCCACCTCTCC & GTTTTTGGTTAGACCGCCACA \\
Klf8 & TCAGAAAGTGGTTCGATGCAG & AACAGAGCTGGGTTCTCCATT \\
Zkscan2 & TGAACCCTCAGATCGACGTG & CTGAAGCATTTACGGAAGGACT \\
Glis1 & GAGGCACTTTCGGACAAGAGG & GAGGCACTTTCGGACAAGAGG \\
Zfp366 & TTCGCAGTTTCGGTATGAACC & GGTGAGTGTAAGGCACCTTGG \\
Gfi1 & GCCTCAGATGACCAGGGGA & TGTTTGGACCCTCGGATACTC \\
Zic2 & CAAGGTCCGGGTGCTTACC & ATTAAAGGGAGGCCCCGAATA \\
Mecom & AAGTAATGAGTGTGCCTATGGC & AGTTGACTCTCGAAGCTCAAA \\
\hline
\end{tabular}

RNA extraction and $m R N A$ sequencing

Total RNA was extracted from the damaged and undamaged utricles using TRIzol reagent (Invitrogen, Carlsbad, CA, USA). The concentrations and purities of the RNA samples were analyzed on a NanoDrop spectrophotometer (Isogen Life Science). All samples had an RNA integrity number value $>8.0$, and an optical density 260:280 ratio >1.9. The rRNA was removed, and mRNA was enriched using magnetic beads with oligo (dT) and then randomly fragmented using fragmentation buffer. The mRNA was used as a template to synthesize the first-strand cDNA using 1st Strand Enzyme Mix (Vazyme). The second-strand cDNA was synthesized using 2nd Strand Marking Buffer and 2nd Strand/End Repair Enzyme Mix (Vazyme). The products were purified by VAHTSTM DNA Clean Beads and then the end of the double strand was repaired and A-tailed. An adapter was joined to the A-tailed products using ligation mix. Suitably sized fragments were selected using VAHTSTM DNA Clean Beads to construct the cDNA library by PCR. The RNA sequencing was then performed using Illumina XTen (Vazyme Biotech Co., Ltd) according to the standard sequencing protocol provided by Illumina (Illumina Inc, USA). Manipulations of raw sequencing data, reads mapping and determination of differentially expressed mRNA were all performed according to the manufacturer's instructions. The differentially expressed mRNAs were identified with q value $\leq 0.05$ and a fold-change of $\geq 2$ between two samples.

Gene ontology and Kyoto Encyclopedia of Genes and Genomes analysis

Different sets of differentially expressed genes were taken as inputs for DAVID Bioinformatics Resource. Kyoto Encyclopedia of Genes and Genomes (KEGG) pathways with EASE value $\leq 0.05$ ( $p$-value from modified fisher exact test, more information can be found in http://david.abcc.ncifcrf.gov/) were deemed to be statistically significantly deviated from the expected distribution, and thus the corresponding pathways were enriched with deregulated genes in question. Considering the huge amount and complex branch structure of gene ontology(GO) biological processes, we used a significant threshold $P$-value adjusted by Benjamini of 0.05 for biological process terms to control the false discovery rate.

\section{qRT-PCR analysis}

The total RNA was extracted by Trizol reagent (Invitrogen, Carlsbad, CA, USA) and then Reverse Transcription System Kit (Takara, Dalian, China) was used to synthesize the first-strand from $1 \mu \mathrm{g}$ of total RNA. The RNA levels were measured using a real-time quantitative PCR system (SYBRPremix Ex Taq kit, Takara, Dalian, China, ABI 7500 Real-Time PCR System, Applied Biosystems, CA, USA). Each assay was performed in triplicate, and $\beta$-actin was used as an endogenous control gene. The primers used were in Table 1. 


\section{Cellular Physiology Cell Physiol Biochem 2018;51:1437-1447

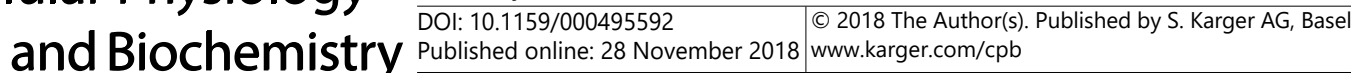

\section{Results}

Hair cells regeneration in neonatal mouse utricle after neomycin damage

In order to confirm that neomycin treatment of neonatal mouse utricle can effectively induce hair cell damage and regeneration, postnatal day (P)2 mouse utricles were dissected out and treated with $3 \mathrm{mM}$ neomycin. After staining with antibodies against Myo7a and Sox2, the cell proliferation marker EdU was administered. In the neomycin-treated utricles, there were much more EdU+ /Sox2+ cells (Fig. 1) compared with the untreated-utricles, indicating that hair cell regeneration were indeed induced in neonatal mouse utricles being treated with neomycin.

\section{Sequencing results and quality control}

A total of 188, 197, 652 raw reads were produced from the 2 cDNA libraries. After quality control, 187, 061, 664 clean reads were obtained. The average GC content of the clean reads of the 2 libraries was $49.33 \%$. The clean reads were aligned against the Mus musculus reference genome. Among these mapped reads, $84.11 \%$ of reads were mapped to exon regions, $12.20 \%$ of reads were mapped to intron regions, and $3.69 \%$ of reads were mapped to intergenic regions. We used Cufflinks to perform quantitative analysis of gene expression, and it was calculated using FPKM (expected number of Fragments Per Kilobase of transcript sequence per Millions base pairs sequenced). The FPKM density distribution was shown in Fig. 2A and B and the Pearson's correlation between samples was shown in Fig. 2C.

\section{Snapshot of mRNAs profiling results generated in neomycin-treated utricles}

Compared to the matched normal controls, a total of 926 mRNAs were significantly differentially expressed (absolute value of $\log _{2}$ Ratio $\geq 1$, q-value $\mid$ FDR $\leq 0.05$ ) in neomycintreated utricles. Methods for determining differential expression was described in Materials and Methods, and the full lists of deregulated mRNAs can be found in Table S1 (For all supplemental material see www.karger.com/10.1159/000495592/). As shown in Fig. 2D, there were 302 and 624 genes that were detected to be over-expressed and down-regulated

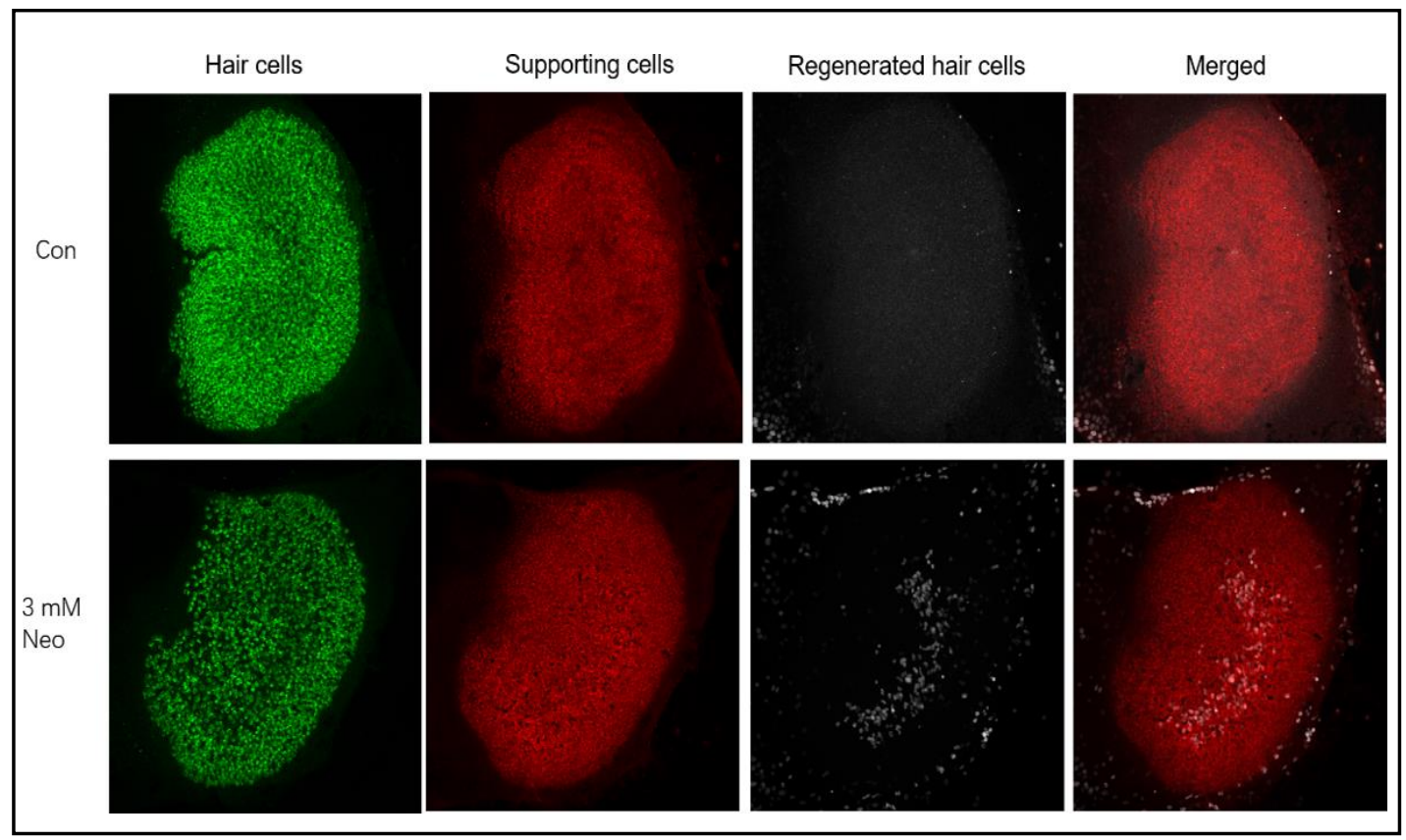

Fig. 1. Hair cells regeneration in neonatal mouse utricle after neomycin damage. The neomycin-treated mouse utricles showed some EdU+ /Sox2+ cells. Green cells represented hair cells, red cells represented supporting cells, and gray cells were EdU+ /Sox $2+$ cells. 


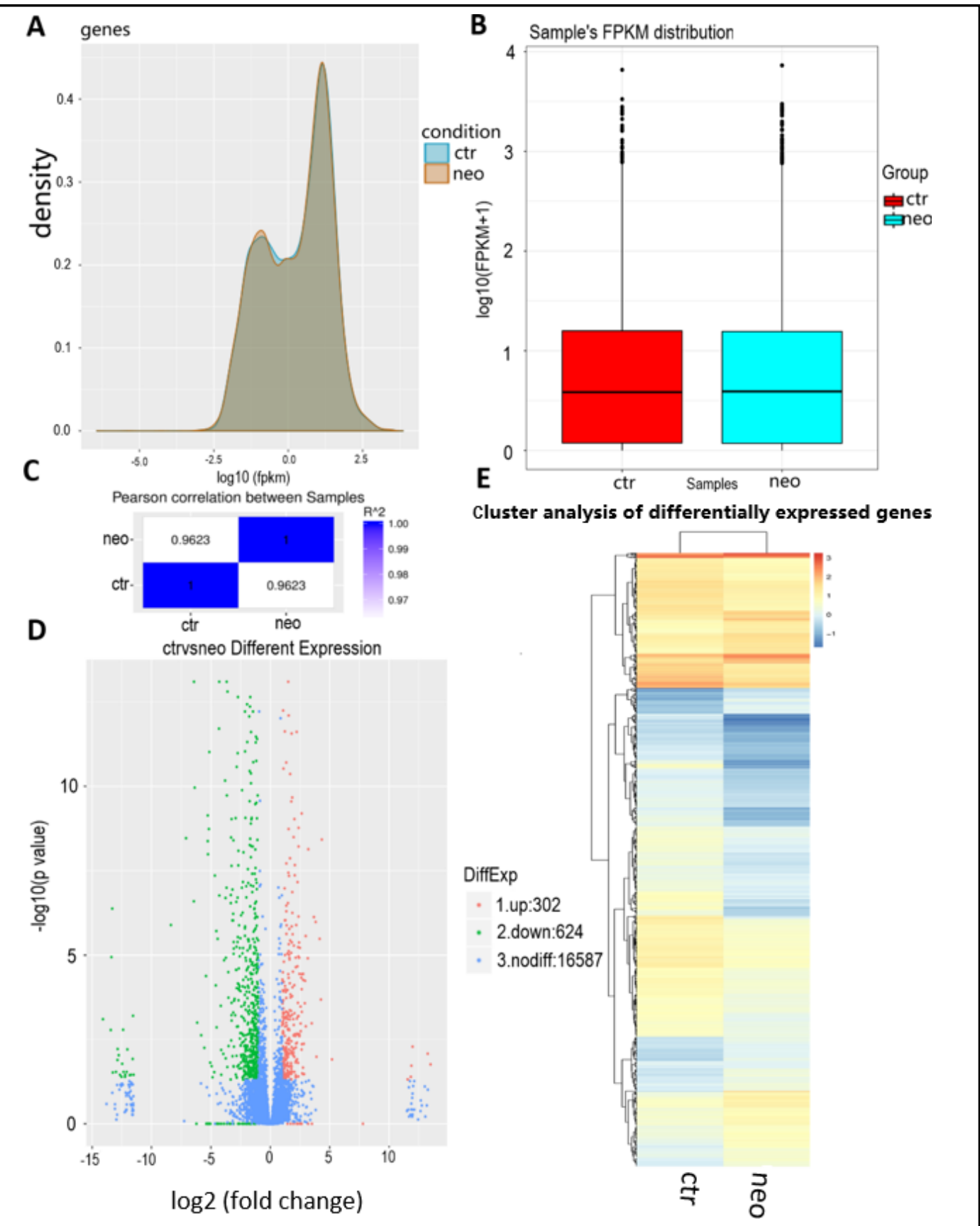

Fig. 2. Differentially expressed genes in neonatal mouse utricle after neomycin injury. (A) Gene expression was shown by a density profile. The $\mathrm{x}$-axis is $\log 10$ (FPKM) and the $\mathrm{y}$-axis is the density of the gene. (B) Gene expression as shown in the FPKM box plot. The $\mathrm{x}$-axis is the sample name, the $\mathrm{y}$-axis is log 10 $(\mathrm{FPKM}+1)$. The box plot for each region has five statistics (top to bottom: maximum, upper quartile, median, lower quartile and minimum). (C) Sample correlation analysis. When the correlation coefficient is close to 1 , it indicates that the similarity of expression patterns between samples is high. (D) The overall profile of differentially expressed genes was revealed by the volcano map. The deregulated genes were indicated by red dots (up-regulation) and green dots (down-regulation). Genes with no significant differential expression were represented by blue dots. The $\mathrm{x}$-axis represents fold change of genes in different samples, and the $y$-axis represents the statistical significance of the gene expression changes. (E) Cluster analysis of expression patterns of differentially expressed genes. The hierarchical clustering results were shown by heat map. Different expression changes were represented by different colors. 


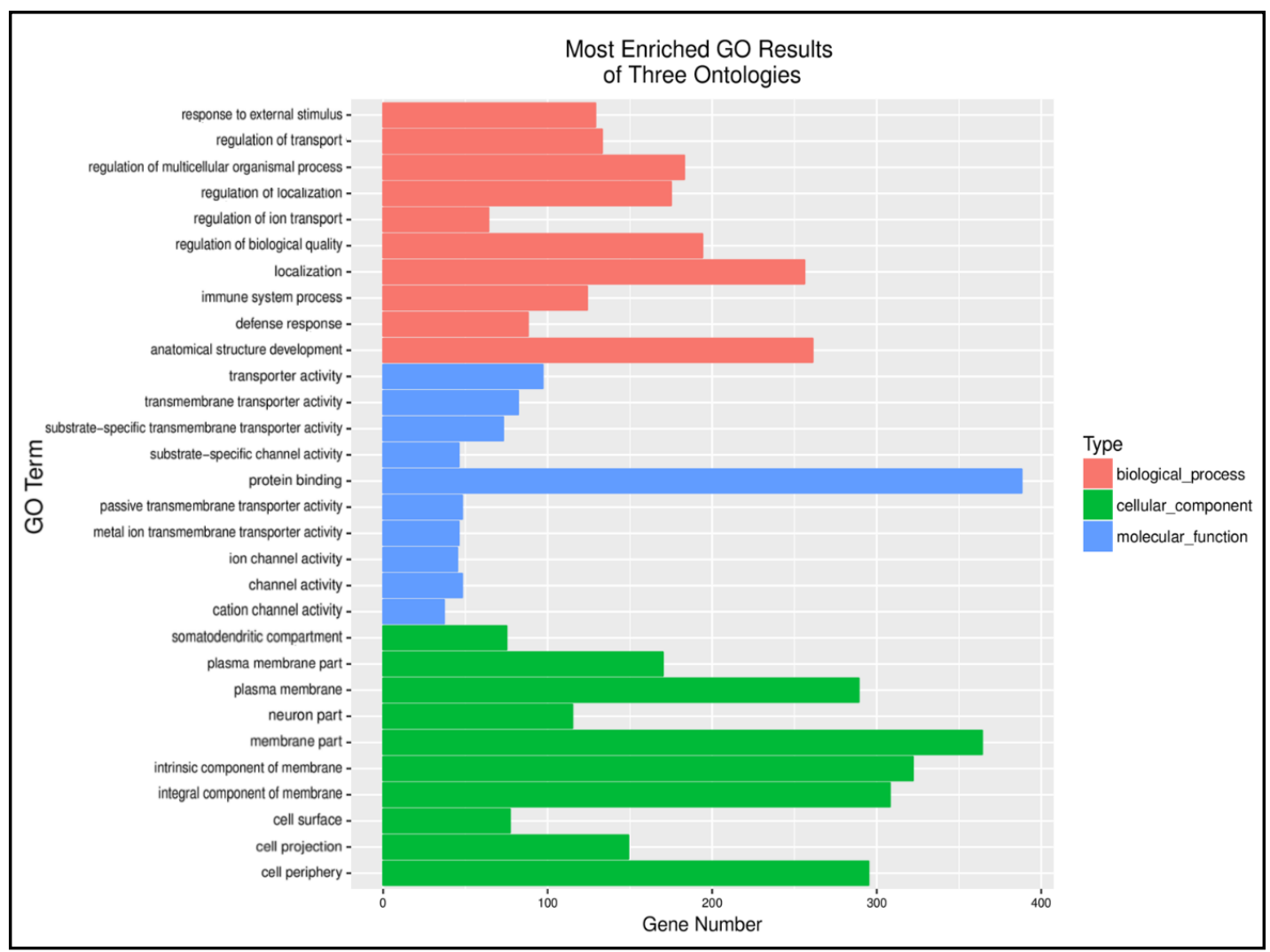

Fig. 3. GO terms significantly enriched with differentially expressed genes. GO analysis of genes differentially expressed after neomycin damage. Different colors were used to distinguish among biological processes, cellular components, and molecular functions.

in neomycin-treated samples. We performed K-means clustering of all differentially expressed genes using the Euclidean distance method associated with complete linkage (Fig. 2E). Several main clusters were plotted with expression patterns of genes involved.

Gene ontology (GO) and pathway analyses of genes significantly deregulated in neomycintreated utricles

All genes that were deregulated in neomycin-treated utricles were then subjected to GO and pathway analyses to identify the significantly deregulated cellular components, molecular functions, biological processes and signaling pathways. As illustrated in Fig. 3, GO analysis of the 926 genes showed that the anatomical structure development, localization, and regulation of biological quality were the most abundant terms in the biological process category. The top three cellular component terms were protein binding, transporter activity and transmembrane transporter activity. The molecular function results showed that membrane part, intrinsic component of membrane, and integral component of membrane were significantly enriched. Interestingly, these terms seem to be related to the natural functions of hair cells. The full lists of GO terms can be found in Table S2. The signaling pathways that were affected in neomycin-treated utricles were also illustrated in Fig. 4. The top 20 pathways included cell adhesion molecules (CAMs), proximal tubule bicarbonate reclamation, rheumatoid arthritis, chemokine signaling pathway, collecting duct acid secretion, osteoclast differentiation, hypertrophic cardiomyopathy (HCM), dilated cardiomyopathy, cardiac muscle contraction, pancreatic secretion, bile secretion, salivary secretion, cytokine-cytokine receptor interaction, gastric acid secretion, type II diabetes mellitus, NF-kappa B signaling pathway, salmonella infection, NOD-like receptor signaling pathway and ECM-receptor interaction. The full lists of KEGG pathways can be found in Table 


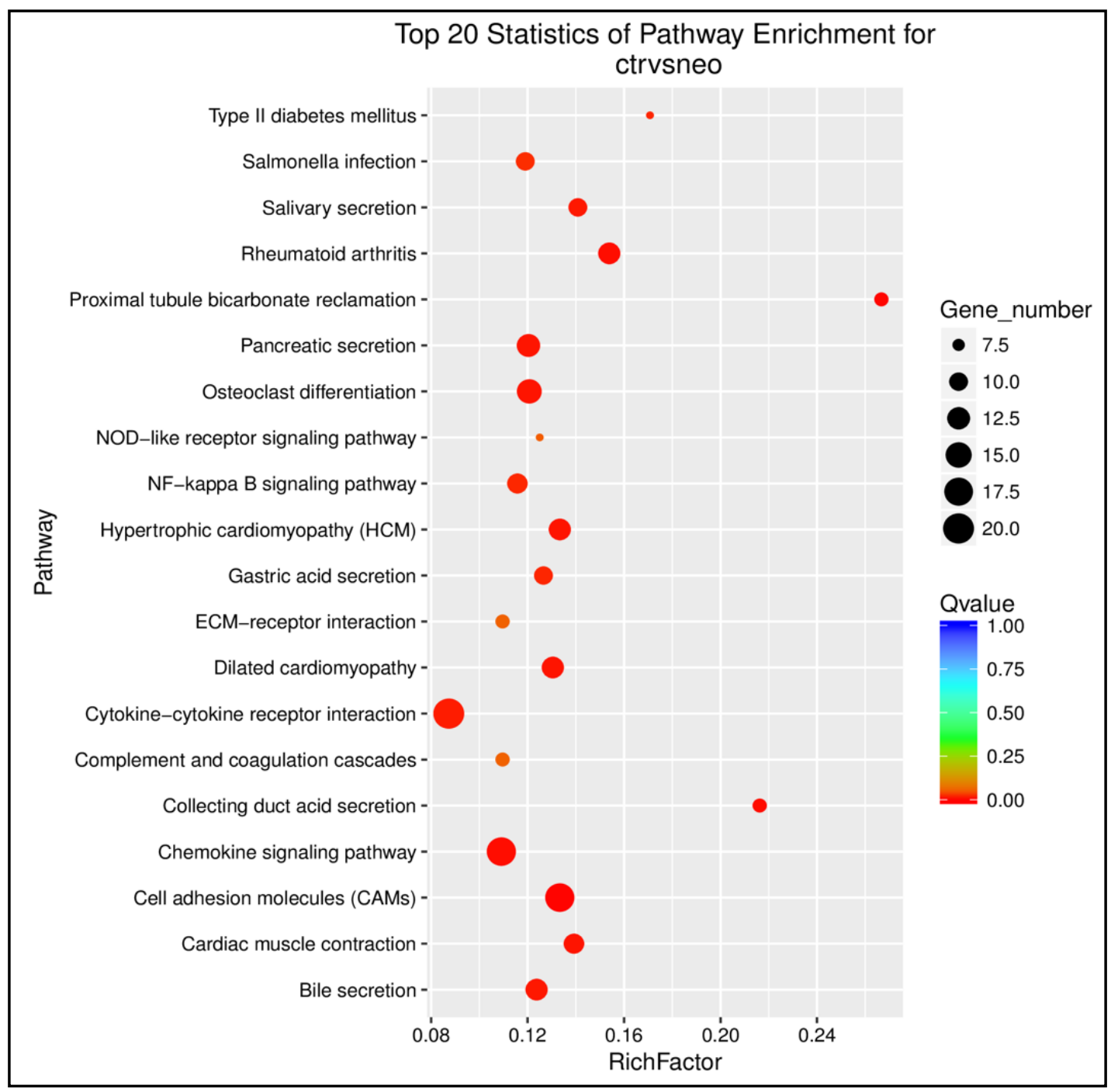

Fig. 4. KEGG pathways significantly enriched with differentially expressed genes. KEGG pathways significantly enriched $(\mathrm{P} \leq 0.05)$ with differentially expressed genes are illustrated. The vertical axis represents the pathway name, and the horizontal axis represents the RichFactor. The size of the point indicates the number of differentially expressed genes in the pathway, and the color of the point corresponds to a different $Q$ value range.

S3. Among these signaling pathways, we focused the NF-kB pathway, as previous studies have reported that this pathway plays an important role in the inflammatory response [14]. Therefore, this pathway is likely to be abnormally activated during the drug-induced damage and subsequent regeneration of hair cells. In addition, we also focused on the Wnt signaling pathway, which was found to have a certain correlation with hair cell regeneration in previous studies [15], although its change was not very significant in our statistical results. Differentially expressed genes involved in these two signaling pathways were shown in Supplementary Fig. S1A and B. 


\section{Cellular Physiology Cell Physiol Biochem 2018;51:1437-1447

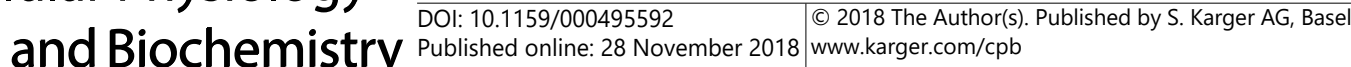 \\ Han et al.: Transcriptome of Hair Cell Regeneration}

\section{Validation of deregulated transcription factors by qRT-PCR}

We inferred that changes in the activity of signaling pathways were caused by abnormal regulation of transcription factors. We then searched transcription factors in differentially expressed genes and classified them according to different families. The classification of transcription factors were shown in Fig. 5A. The full lists of different families of transcription factors can be found in Table S4. There were 9 differentially expressed genes in the transcription factors from the zf- $\mathrm{C} 2 \mathrm{H} 2$ family. The zf-C2H2 proteins act by directly binding to DNA to control nearby genes. Previous studies showed that zf-C2H2 are likely to be associated with multiple physiological processes and stress responses [16]. qRT-PCR was then carried out to validate the differentially expressed genes involved in the zf-C2H2 family. The qRT-PCR results for all of the deregulated transcription factors were analyzed statistically using t-test. The results showed that the expression patterns of these genes were in excellent agreement with the RNA-seq results (Fig. 5B). This indicates that these transcription factors indeed expressed abnormally during hair cell injury and subsequent regeneration.

\section{Discussion}

Hearing and balance deficits often affect humans permanently, as our ears stop producing hair cells within a few days after birth. However, production occurs throughout life in the ears of numerous non-mammalian species $[5,6]$. The reasons that prevents hair cell regeneration in mammals have been studied for many years, and it has become clear that some deregulated cellular signals in mammalian ears should contribute to putting the brakes on regeneration.

In this study, we found 926 genes that were expressed at significantly different levels in neomycin-treated neonatal mouse utricle. The majority of the differentially expressed genes have not been reported in the inner ear before, while some of them have been studied in previous reports. The biological functions of many differentially expressed genes and their signaling pathways may be related to the biological characteristics of hair cells. In GO analysis, we have seen many terms that are directly related to hair cell function, such as anatomical structure development, transmembrane transporter activity and intrinsic component of membrane. The auditory function of inner ear is correlated with the anatomical structure [17]. A transporter-like protein usually acts as a piezoelectric transducer that mediates the fast electromotility of outer hair cells required for auditory acuity in mammals [18]. The intrinsic membrane filtering of inner hair cells ensures accurate encoding of the phasic or sustained components of the cell's in vivo receptor potential, crucial for sound localization and ultimately survival [19]. In KEGG pathway analysis, there were also many signaling pathways that have been reported to be correlated with hair cell function, such as cell adhesion molecules (CAMs) and NF-kappa B signaling pathway. Some adhesion molecules have been found to play a key role in inner ear and hair cell development, including notch and its ligands [20]. It has been demonstrated that the transcription factor NF-kappaB is required for survival of immature auditory hair cells in corti [21]. From our own results, we also found that NF-kappaB may be correlated with the survival of hair cells in utricle. More importantly, we found and validated that 9 transcription factors from the zf-C2H2 family were differentially expressed in neomycin-treated neonatal mouse utricle. More interestingly, eight of the nine genes were down-regulated. This family of transcription factors has long been thought to be involved in cell development [16]. Further research is needed to investigate their functions and regulatory mechanisms. Now that the CRISPR technique has been well developed. It may be used to regenerate hair cells by simultaneous regulation of these transcription factors. 


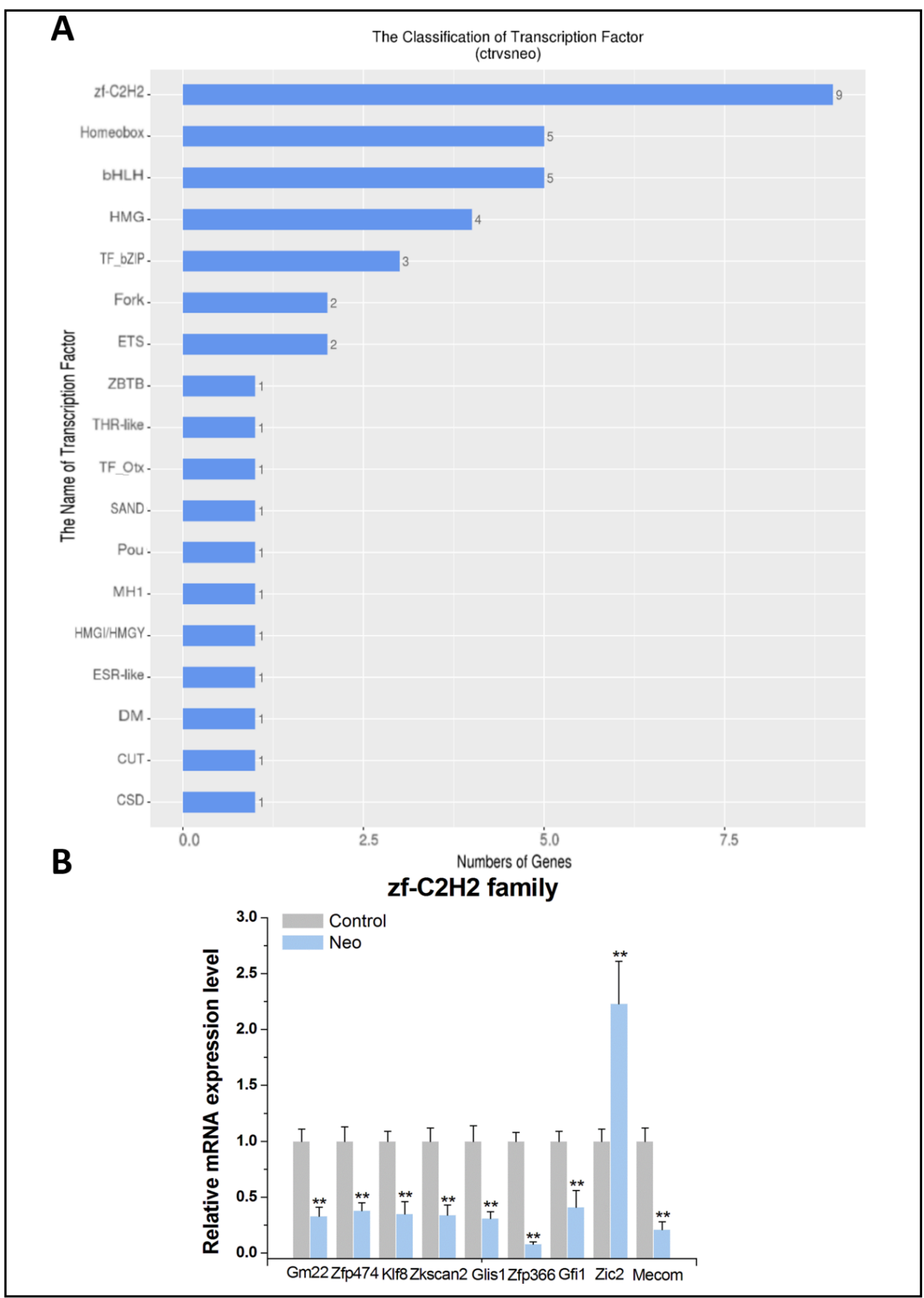

Fig. 5. Differentially expressed transcription factors in neonatal mouse utricle after neomycin injury. (A) The differentially expressed transcription factors from various families. (B) Quantitative RT-PCR analysis of the nine transcription factors that are differentially expressed after neomycin injury as identified by RNASeq. Data are presented as the relative fold change in expression. Student's paired t-test; ${ }^{* *} \mathrm{p}<0.01$. 


\section{Cellular Physiology Cell Physiol Biochem 2018;51:1437-1447 and Biochemistry \begin{tabular}{l|l} 
DOI: 10.1159/000495592 2018 The Author(s). Published by S. Karger AG, Basel \\
Published
\end{tabular}

\section{Conclusion}

In summary, here we presented a comparative analysis of the whole-genome expression profiles of mRNA in neomycin-treated neonatal mouse utricle. Our findings revealed the common changes in a few conserved biological processes or pathways in human diseases and highlighted the effects of several commonly deregulated genes on hair cell regeneration. Taken together, our study can serve as a valuable source for future studies on hair cell damage / regeneration and some of the highlighted genes, pathways or processes may be useful for therapeutic purposes.

\section{Acknowledgements}

We are indebted to the participants, whose names were not included in the author list, but who contributed to this work. Conceived and designed the experiments: YL GN. Performed the experiments: JH HW. Analyzed the data: YL. Contributed reagents/materials/analysis tools: YL GN. Wrote the paper: YL. Designed the project and gave final approval of the version to be published: YL GN. This work was funded by the Shenzhen public service platform for vertigo diagnosis \& treatment and vestibular function remodeling (s2016005470013) and the Sanming Project of Medicine in Shenzhen (SZSM201612031).

\section{Disclosure Statement}

The authors declare that there are no conflicts of interest.

\section{References}

1 Bermingham-McDonogh 0, Rubel EW: Hair cell regeneration: winging our way towards a sound future. Curr Opin Neurol 2003;13:119-126.

2 Wu Z, Grillet N, Zhao B, Cunningham C, Harkins-Perry S, Coste B, Ranade S, Zebarjadi N, Beurg M, Fettiplace R, Patapoutian A, Mueller U: Mechanosensory hair cells express two molecularly distinct mechanotransduction channels. Nat Neurosci 2017;20:24-33.

- Furness DN: Molecular basis of hair cell loss. Cell and tissue research 2015;361:387-399.

4 Smith ME, Groves AK, Coffin AB: Sensory Hair Cell Death and Regeneration. Front Cell Neurosci 2016;10:208.

5 Kniss JS, Jiang L, Piotrowski T: Insights into sensory hair cell regeneration from the zebrafish lateral line. Curr Opin Genet Dev 2016;40:32-40.

-6 Xu J, Ueno H, Xu CY, Chen B, Weissman IL, Xu PX: Identification of mouse cochlear progenitors that develop hair and supporting cells in the organ of Corti. Nat Commun 2017;8:15046.

7 Bramhall NF, Shi F, Arnold K, Hochedlinger K, Edge AS: Lgr5-positive supporting cells generate new hair cells in the postnatal cochlea. Stem Cell Reports 2014;2:311-322.

-8 Franco B, Malgrange B: Concise Review: Regeneration in Mammalian Cochlea Hair Cells: Help from Supporting Cells Transdifferentiation. Stem Cells 2017;35:551-556.

-9 Jiang L, Xu J, Jin R, Bai H, Zhang M, Yang S, Zhang X, Zhang X, Han Z, Zeng S: Transcriptomic analysis of chicken cochleae after gentamicin damage and the involvement of four signaling pathways (Notch, FGF, Wnt and BMP) in hair cell regeneration. Hear Res 2018;361:66-79.

10 Żak M, Klis SFL, Grolman W: The Wnt and Notch signalling pathways in the developing cochlea: formation of hair cells and induction of regenerative potential. Int J Dev Neurosci 2015;47:247-258.

11 Burns JC, Corwin JT: A historical to present-day account of efforts to answer the question:"what puts the brakes on mammalian hair cell regeneration?". Hear Res 2013;297:52-67. 


\section{Cellular Physiology Cell Physiol Biochem 2018;51:1437-1447

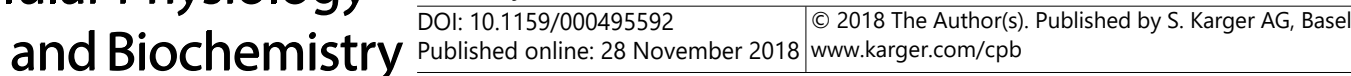

Han et al.: Transcriptome of Hair Cell Regeneration

12 Zhang S, Zhang Y, Yu P, Hu Y, Zhou H, Guo L, Xu X, Zhu X, Waqas M, Qi J, Zhang X, Liu Y, Chen F, Tang M, Qian X, Shi H, Gao X, Chai R: Characterization of Lgr5+ progenitor cell transcriptomes after neomycin injury in the neonatal mouse cochlea. Front Mol Neurosci 2017;10:213.

13 Waqas M, Guo L, Zhang S, Chen Y, Zhang X, Wang L, Tang M, Shi H, Bird PI, Li H, Chai R: Characterization of Lgr5+ progenitor cell transcriptomes in the apical and basal turns of the mouse cochlea. Oncotarget 2016;7:41123.

14 Tak PP, Firestein GS: NF-кB: a key role in inflammatory diseases. J Clin Invest 2001;107:7-11.

15 Greco V, Chen T, Rendl M, Schober M, Pasolli HA, Stokes N, Dela Cruz-Racelis J, Fuchs E: A two-step mechanism for stem cell activation during hair regeneration. Cell Stem Cell 2009;4:155-169.

16 Bass J I F, Sahni N, Shrestha S, Garcia-Gonzalez A, Mori A, Bhat N, Yi S, Hill DE, Vidal M, Walhout AJM: Human gene-centered transcription factor networks for enhancers and disease variants. Cell 2015;161: 661-673.

17 Bibas AG, Xenellis J, Michaels L, Anagnostopoulou S, Ferekidis E, Wright A: Temporal bone study of development of the organ of Corti: correlation between auditory function and anatomical structure. J Laryngol Otol 2008;122:336-342.

18 Schaechinger TJ, Gorbunov D, Halaszovich CR, Moser T, Kügler S, Fakler B, Oliver D: A synthetic prestin reveals protein domains and molecular operation of outer hair cell piezoelectricity. EMBO J 2011;30:27932804.

19 Johnson SL: Membrane properties specialize mammalian inner hair cells for frequency or intensity encoding. Elife 2015;4: e08177.

20 Kelley MW: Cell adhesion molecules during inner ear and hair cell development, including notch and its ligands. Curr Top Dev Biol 2003;57:321-356.

21 Nagy I, Caelers A: Monge A, Bonabi S, Huber AM, Bodmer D. NF-kappaB-dependent apoptotic hair cell death in the auditory system. Audiol Neurootol 2007;12:209-220. 